\title{
Bidirectional histone-gene promoters in Aspergillus: characterization and application for multi-gene expression
}

\author{
Jakob K. H. Rendsvig, Christopher T. Workman and Jakob B. Hoof (i)
}

\begin{abstract}
Background: Filamentous fungi are important producers of enzymes and bioactive secondary metabolites and are exploited for industrial purposes. Expression and characterization of biosynthetic pathways requires stable expression of multiple genes in the production host. Fungal promoters are indispensable for the accomplishment of this task, and libraries of promoters that show functionality across diverse fungal species facilitate synthetic biology approaches, pathway expression, and cell-factory construction.

Results: In this study, we characterized the intergenic region between the genes encoding histones $\mathrm{H} 4.1$ and $\mathrm{H} 3$, from five phylogenetically diverse species of Aspergillus, as bidirectional promoters (Ph4h3). By expression of the genes encoding fluorescent proteins mRFP1 and mCitrine, we show at the translational and transcriptional level that this region from diverse species is applicable as strong and constitutive bidirectional promoters in Aspergillus nidulans. Bioinformatic analysis showed that the divergent gene orientation of $h 4.1$ and $h 3$ appears maintained among fungi, and that the Ph4h3 display conserved DNA motifs among the investigated 85 Aspergilli. Two of the heterologous Ph4h3s were utilized for single-locus expression of four genes from the putative malformin producing pathway from Aspergillus brasiliensis in A. nidulans. Strikingly, heterologous expression of mIfA encoding the non-ribosomal peptide synthetase is sufficient for biosynthesis of malformins in A. nidulans, which indicates an iterative use of one adenylation domain in the enzyme. However, this resulted in highly stressed colonies, which was reverted to a healthy phenotype by co-expressing the residual four genes from the putative biosynthetic gene cluster.
\end{abstract}

Conclusions: Our study has documented that Ph4h3 is a strong constitutive bidirectional promoter and a valuable new addition to the genetic toolbox of at least the genus Aspergillus.

Keywords: Aspergillus, Bidirectional promoters, Histone, Motifs, Heterologous expression, Biosynthetic gene clusters, Malformin, Non-ribosomal peptide synthetase, Adenylation domain

\section{Background}

Certain species of filamentous fungi particularly of genus Aspergillus are widely applied for production of primary metabolites (organic acids), industrial enzymes, pharmaceutical proteins, and bioactive secondary metabolites (SMs). Regardless of the product type and the origin, the availability of suitable fungal promoters is an important consideration for controlling gene expression in new

*Correspondence: jblni@dtu.dk

Department of Biotechnology and Biomedicine, Technical University of Denmark, 2800 Kongens Lyngby, Denmark hosts. For this reason, characterization of constitutive or inducible promoters that function in a heterologous context increases the application range of filamentous fungi as expression hosts. Researchers in the last few decades have identified a small number of promoters that have become de facto standards for expression in Aspergillus, most notably the constitutive PgpdA [1] and Ptef1 [2], the inducible PalcA [3] and PglaA [4], and the synthetic Tet-on expression system [5]. While sufficient for simple gene-expression tasks, this promoter library may become limiting when applied to biosynthetic pathways with multiple genes. Moreover, heterologous promoters 
that provide a similar transcriptional strength to endogenous promoters are attractive, as they reduce the risk of integrating the expression cassettes in the locus of an endogenously derived promoter.

Heterologous expression of SM pathways is important for elucidation of biosynthetic pathways originating from non-tractable hosts or for successful activation of a silent pathway [6]. Hence, it requires the expression of all pathway-contributing genes, which are often organized as a physically coherent biosynthetic gene cluster (BGC). The expression of the full BGC in the native or heterologous host may be accomplished by overexpressing a gene-cluster specific transcriptional activator associated with the BGC [7-9]. However, a significant number of BGCs do not contain a gene encoding a transcription factor (TF) and only $\sim 30 \%$ of the gene clusters display co-expression with contiguous TFs [10]. In addition, the overexpression of activating TFs may have the drawback of an indirect activation of other BGCs compromising the analysis of the specific gene cluster under investigation. An alternative approach to ensure activation of the BGC is by the functional expression of each gene. This requires multiple promoters reacting to the same stimuli, since consecutive use of the same promoter would be prone to genetic instability and genome defense mechanisms like repeatinduced point mutations [11].

Therefore, we set out to exploit the reservoir of stable bidirectional promoters, which would ease the heterologous expression of BGCs. One example is the histones $\mathrm{H} 4.1$ and $\mathrm{H} 3$ encoded by the genes $h 4.1$ and $h 3$. These genes are divergently transcribed and the intergenic region therefore likely serves as a bidirectional promoter (Ph4h3). Moreover, in filamentous fungi the orientation of these genes appear conserved and are most often present as single copies in the genome, separated by $799 \mathrm{bp}$ in the case of Aspergillus nidulans. The Ph4h3 of A. nidulans ( $h 4.1 ; h h f A$ and $h 3$; $h h t A$ ) was first characterized by Ehinger et al. [12], who showed that the expression from each of the respective ends were approximately equal and regulated in accordance with the cell cycle. This was also observed for both copies of h4.1 (HHF1, HHF2) and $h 3$ (HHT1, HHT2) in the yeast Saccharomyces cerevisiae $[13,14]$. The endogenous promoters of histone encoding genes have previously been used for various purposes in Penicillium funiculosum [15], Aspergillus oryzae [16], and the yeast Arxula adeninivorans [17]. Recently, the Ph4h3 from the yeast Pichia pastoris was used to generate a library of synthetic bidirectional promoters [18]. The use of other natural bidirectional promoters in filamentous fungi has so far been ascribed to the inducible promoters controlling expression of $p c b A B / p c b C$ from Penicillium chrysogenum [19], niaD/niiA and stcA/stcB from $A$. nidulans [20].
Owing to the great importance of $\mathrm{H} 4.1$ and $\mathrm{H} 3$ proteins in chromatin structure and the apparent widespread conservancy in the divergent orientation, we hypothesized that $\mathrm{P} h 4 \mathrm{~h} 3$ from different species would be functional across species within a fungal genus. Moreover, we aimed at investigating if the employment of multiple $\mathrm{Ph} 4 \mathrm{~h} 3 \mathrm{~s}$ of different species could be applied for one-locus expression of heterologous BGCs. To accomplish this, we expressed the two genes encoding fluorescent proteins mRFP1 and mCitrine in $A$. nidulans using five different Ph4h3s; the endogenous $A$. nidulans $\mathrm{Ph} 4 \mathrm{~h} 3$, and the heterologous Ph4h3s from Aspergillus niger, Aspergillus flavus, Aspergillus clavatus, and Aspergillus terreus. These five species represent five different sections of genus Aspergillus; Nidulantes, Nigri, Flavi, Clavati, and Terrei, and therefore represent a phylogenetic set of heterologous promoters applied for expression analysis. The relative expression strength of both ends of each promoter was qualitatively accessed by fluorescence microscopy and quantitatively analyzed by RT-qPCR during solidstate and submerged cultivations at different growth phases. Lastly, the Ph4h3 of A. niger and A. clavatus were applied for heterologous expression of the putative BGC responsible for biosynthesis of the malformin compound family from $A$. brasiliensis in A. nidulans [21].

\section{Results}

\section{Sequence analysis of Aspergillus Ph4h3 promoters}

The DNA sequences from orthologous intergenic regions of $h 4.1$ and $h 3$ (Ph4h3) were collected from 85 genome sequenced Aspergillus species and five fungi representing other important fungal genera; Neurospora crassa, Trichoderma reesei, Penicillium rubens, Agaricus bisporus, and S. cerevisiae for sequence comparison. The $h 4.1$ and $h 3$ genes of each species were inferred from protein sequence homology to $h h f A$ ( $h 4.1$, in this study $h 4$ for simplicity) and hhtA (h3) of $A$. nidulans using BLASTp [22]. The extracted $\mathrm{Ph} 4 h 3$ sequences and gene prediction details are listed in Additional file 1: Table S1, and further described in Additional file 2.

In brief, the 85 curated Aspergillus Ph4h3 sequences were analyzed to generate a multiple sequence alignment with a 790 bp consensus sequence. The alignment revealed several highly conserved DNA motifs, located predominantly in the region -100 to $-200 \mathrm{nt}$ relative to the translational start site (ATG) at each end of the bidirectional promoters (Additional file 2: Figure S2). The consensus sequence was scanned by 177 fungal TF binding motif models from JASPAR [23], resulting in 81 matches (52 different TFs) with a score above 9 (Additional file 3: Tables S3-S4).

In the search for regulatory motifs involved in the bidirectional expression, we primarily focused on binding 
sites with two or more putative matches in reverse orientation located in opposite ends of the Ph4h3 consensus sequence. In addition, we included regulatory motifs described to be involved in histone gene expressions in humans and S. cerevisiae [24-26]. Firstly, expression of core histone-encoding genes in $S$. cerevisiae occurs primarily during $\mathrm{S}$-phase activated through the DNA binding of Spt10 and subsequent recruitment of S-phasespecific Spt21 [27, 28]. Analysis of the consensus Ph4h3 sequence for presence of the yeast Spt10 motif [29] (retrieved from YeTFaSCo; motif \#1322) revealed four putative Spt10 binding sites ordered pairwise (separated by 63 and $59 \mathrm{nt}$ ) in opposite orientation in each end of the promoter (Fig. 1), as also reported for S. cerevisiae [25]. Orthologues of yeast Spt10 were identified by protein sequence homology in each of the 85 Aspergilli $(<2 \mathrm{E}-53)$ (data not shown). Secondly, the negative cell cycle regulation of the gene expression partly derives from the NEG region and associated motif [26,30]. Analysis of the consensus Ph4h3 sequence with the NEG motif [14] revealed three low-scoring matches (Fig. 1), positioned similarly as in S. cerevisiae [30]. Thirdly, the NF-Y complex regulates core histone gene expression in humans via the motif 5'-CCAAT-3' [24], and interestingly we found two pairs of HAP3/HAP5 motifs (homologs of human NFY $\mathrm{B}$ and $\mathrm{C}$ ) in the consensus $\mathrm{Ph} 4 \mathrm{~h} 3$ oriented towards the promoter ends (Fig. 1). Additionally, two putative binding sites for yeast MIG1-3 (fungal CreA) [31] were found located in opposite ends and in reverse orientations in the consensus Ph4h3 (Fig. 1). Lastly, the analysis also revealed several lower scoring motifs of the major G1/S cell-cycle-dependent transcription factor MBF (MluI cell cycle box binding factor) [32], which is involved in the positive regulation of histone gene transcription during the G1/S transition of the mitotic cell cycle in S. cerevisiae [33] (Additional file 3). In addition to the identified putative motifs of known TFs, the alignment of Ph4h3 revealed several highly conserved motifs that could not be associated with a given TF in our analysis, among others; $5^{\prime}$-TACAAATA- $3^{\prime}$ resembling a TATA box, and $5^{\prime}$-CTCGCTTA-3' (Additional file 2: Figure S2).

The DNA motifs discovered in $\mathrm{P} h 4 h 3$ of Aspergilli could partially be found in the Ph4h3 of the four selected reference filamentous fungi $N$. crassa, $T$. reesei, P. rubens, and A. bisporus (Additional file 3). Collectively, we found DNA motifs in the Ph4h3 of Aspergilli from both higher eukaryotes and yeasts, potentiating shared regulatory features. Interestingly, overrepresentations of putative TF binding sites are located in the promoter region on the coding strand upstream of each histone gene (Fig. 1). Here we have presented an overview of conserved regulatory DNA motifs in the Ph4h3 of Aspergillus, and the dataset of $\mathrm{P} h 4 h 3$ sequences provided in this study may spur more investigations.

\section{Design of strains expressing reporter genes using the bidirectional promoters}

The Ph4h3 of five Aspergilli; A. nidulans (NID), A. niger (NIG), A flavus (FLA), A. clavatus (CLA), and A. terreus (TER), were chosen as the proof of concept promoters employed to express genes encoding the fluorescent proteins $\mathrm{mRFP}$ and mCitrine, with transcriptional termination by Ttef 1 and TtrpC, respectively. The promoters were defined as the intergenic regions between the genes for $\mathrm{H} 4.1$ and $\mathrm{H} 3$, respectively; A. nidulans (AN0734.2 and AN0733.2), A. niger (ANI_1_974074 and ANI_1_976074), A. clavatus (ACLA_021660 and ACLA_021650), A. flavus (AFLA_017620 and AFLA_017610), and A. terreus (ATEG_00587 and ATEG_00586). The Ph4h3s had approximately the same lengths in the five species; $A$. nidulans (799 bp), A. niger (828 bp), A. flavus (821 bp), $A$. clavatus (853 bp), and $A$. terreus (822 bp).

Using the five Ph4h3s, 10 constructs were made to confer expression of mRFP-Ttef1 and mCitrine-TtrpC from either end of each Ph4h3 (Fig. 2). For comparison, two reference constructs were tested using the promoters of $g p d A$ and tef 1 expressing $m R F P$ with transcriptional termination by TtrpC. The PgpdA and Ptef1 from

Fig. 1 Putative regulatory motifs in Ph4h3. The 790 bp consensus Ph4h3 with the putative motif positioning shown and motif $5^{\prime}$-3' directionality
indicated by an arrow. The promoter length and the translational start site (ATG/CAT) at each promoter end are shown. The distribution of
motifs is to scale, while motif lengths are not. The motif sequence found in the consensus Ph4h3 are listed in Additional file 3, and the sequence
conservation of each motif is shown in the Logo-plot in Additional file 2: Figure S2. Note; the two CreA sites positioned downstream of the Spt10
site have four and three overlapping nucleotides, respectively. The two pairs of HAP3/HAP5 sites (15 nt per site) have 10 overlapping nucleotides,
incl. the core CCAAT motif. The two outer most Spt10 motifs are 51 and 58 nt upstream of the respective promoter end




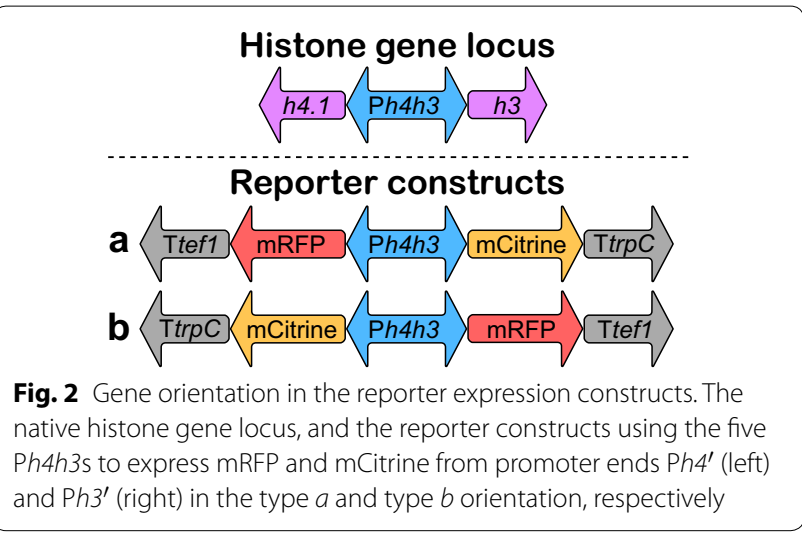

A. nidulans had similar lengths to $\mathrm{Ph} 4 \mathrm{~h} 3$ of $836 \mathrm{bp}$ and 886 bp, respectively.

Each of the 10 Ph $4 h 3$ expression constructs (Fig. 2) and the control constructs with mRFP controlled by PgpdA and Ptef1 were assembled by USER (uracil specific excision reagent) cloning. USER cloning is dependent on the inclusion of uracil-linked $5^{\prime}$ extensions of the primers used for fragment amplification, enabling the generation of complimentary overhangs and seamless assembly of DNA fragments. Verified vectors were linearized prior to transformation and gene targeting was performed in a non-homologous end joining deficient $A$. nidulans host strain targeting the integration site 4 (IS4) [34]. Biological triplicates from each transformation were validated rigorously by diagnostic PCR for correct targeted integration and to consist of homokaryotic nuclei in respect to IS4 (Additional file 4: Figure S3). Potential additional integration events of reporter constructs at the endogenous loci of Ph4h3, TtrpC, and Ttef1 were excluded by diagnostic PCR (Additional file 4). None of the constructed $\mathrm{P} h 4 h 3$ reporter strains showed any morphological change compared to the reference strain (Additional file 4: Figure S4a), and we continued with evaluating the promoter performance.

\section{Evaluation of promoter strength by qualitative fluorescence microscopy}

The 10 promoter constructs using the promoter end $\mathrm{P} h 4^{\prime}$ and $\mathrm{P} h 3^{\prime}$ to drive expression of $m R F P$ were compared to the controls, PgpdA and Ptef1, for relative promoter strength by fluorescence microscopy. Each of the strain types were cultivated in triplicate on agar-covered microscopy slides for $20 \mathrm{~h}$, and exposed single representative leading hyphae are shown in Fig. 3.

Based on the observations presented in Fig. 3, all ten promoter combinations were able to drive $m R F P$ expression as the mRFP production appeared close to equal in strength for all individual types of constructs as well as for the triplicates. Compared to the control-promoter constructs, the mRFP production appeared much stronger in the Ph4h3-based expression strains than in $\operatorname{PgpdA}$, but not as strong as the Ptef1 strains.

An equivalent signal of mCitrine fluorescence was observed from both ends of the five Ph4h3 promoters (data not shown). However, the mCitrine signal was slightly weaker than the mRFP signal in all cases (despite expressed from the same promoter end) and was therefore not used in the promoter comparison. This confirmed that both ends of the bidirectional promoter were active during growth.

\section{Quantitative determination of promoter strength during solid-state cultivation}

The qualitative analysis of promoter strength by protein fluorescence indicated that the bidirectional $\mathrm{Ph} 4 \mathrm{~h} 3$ promoters were of similar strength in both directions, but we also wanted to quantitatively measure the promoter strength at the mRNA level using RT-qPCR. Biomass was harvested during growth on plates $(72 \mathrm{~h}$ ) from seven strains; five strains based on the $\mathrm{P} h 4 h 3$ promoter constructs in the type $a$ orientation expressing both $m R F P$ and $m$ Citrine (Fig. 2) and two strains using the reference

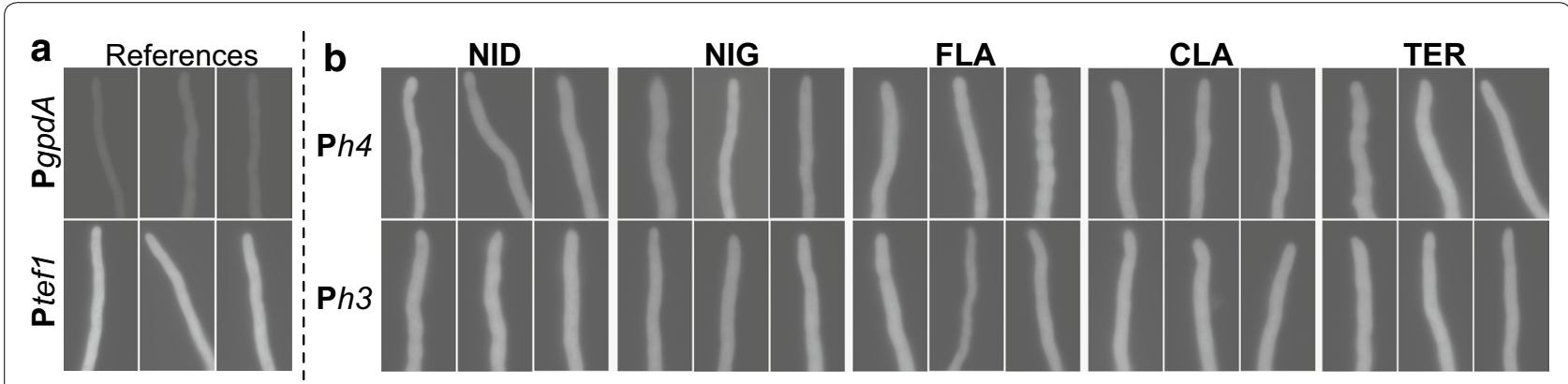

Fig. 3 Fluorescence microscopy of strains expressing mRFP as reporter. Single leading hyphae from biological triplicates of a control promoter strains with PgpdA and Ptef1, or b the ten Ph4h3 based strains, expressing mRFP under the control of either the Ph4' (type a constructs) or Ph3' (type $b$ constructs) of the five Ph4h3 promoters from A. nidulans (NID), A. niger (NIG), A. flavus (FLA), A. clavatus (CLA), and A. terreus (TER) 
promoters, PgpdA and Ptef1, to drive mRFP expression (see Fig. 4).

Firstly, no significant differences were observed between the relative expression levels obtained from the five Ph4h3s of $A$. nidulans (NID), A. niger (NIG), A flavus (FLA), A. clavatus (CLA), and A. terreus (TER), when compared to the endogenous $A$. nidulans $\mathrm{Ph} 4 h 3$. This observation was also true for the different orientations of $m R F P$ and $m$ Citrine, with the exception of the relative expression level of mCitrine $\left(\mathrm{Ph} 3^{\prime}\right)$ from the A. terreus $\mathrm{Ph} 3^{\prime}$, which was significantly higher than $m$ Citrine expressed from both the $\mathrm{P} h 3^{\prime}$ of $A$. clavatus $(P=0.033)$ and A. flavus $(P=0.0048)$. Secondly, PgpdA and Ptef1 indicated a lower and higher expression level of $m R F P$, respectively, compared to the Ph4h3s, however only the $m$ Citrine level expressed from $\mathrm{P} h 3^{\prime}$ of $A$. clavatus was significantly different from Ptef1 $(P=0.0075)$.

$\operatorname{PgpdA}$ is routinely used for various types of expression constructs in $A$. nidulans, and is historically approximately $2.3 \mathrm{kbps}$ long [35]. In this study, we have shortened PgpdA to $836 \mathrm{bps}$ for it to be of comparable size to the other promoters used. Therefore, the influence that this reduction in length may have for the promoter strength was examined by comparing the 836 bps version to the $2.3 \mathrm{kbps}$ long $\operatorname{Pg} p d A\left(\operatorname{Pgpd} A_{2.3}\right)$, both fused to $m R F P-\mathrm{T} t r p C$ (Additional file 4: Figure S5). RT-qPCR did not reveal a significant difference between the two promoter variants.

As described in the bioinformatics analysis, long regions of the Ph4h3 were highly conserved in Aspergilli, notably in the region 100-200 bps upstream of each end. To investigate the importance of the less conserved middle region, two constructs were made with internal truncations of the A. nidulans $\mathrm{Ph} 4 \mathrm{~h} 3$, in which the middle part of the Ph4h3 was removed, specifically the middle $400 \mathrm{bp}$ or $600 \mathrm{bp}$, thereby constructing two shortened versions of $\mathrm{Ph} 4 \mathrm{~h} 3 \mathrm{~s}$ composed of the outermost $200 \mathrm{bp}$ or

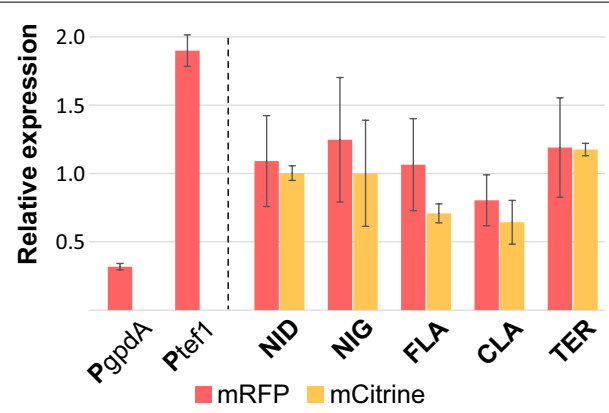

Fig. 4 Relative expression from Ph4h3 during solid state cultivation. Relative expression by RT-qPCR on mRFP (red) and mCitrine (orange) of each of the five Ph3h4s (NID, NIG, FLA, CLA, TER), and the references promoters, PgpdA and Ptef1, see text for details
$100 \mathrm{bp}$ of each end (pAC1688-1689), respectively. After verification for targeted integration in A. nidulans IS4 and homokaryon, the strains were analyzed by fluorescence microscopy. Here, removal of the middle $400 \mathrm{bp}$ from $\mathrm{Ph} 4 h 3$ decreased expression at each end slightly, while removal of the middle $600 \mathrm{bp}$ abolished expression from both ends (data not shown). This may be due to the removal of the inner most Spt10 site in each end through the 600 bp truncation (Fig. 1), as the pairwise arrangement of these motifs was deemed important for the gene activation in yeast [25].

Cultivations on solid medium showed a pattern of mixed expression due to the presence of different tissue types (e.g. hyphae, apex, conidia, phialides, metulae), and hence differentiated stages of cellular dormancy. For this reason, an analysis of $A$. nidulans $\mathrm{Ph} 4 \mathrm{~h} 3$ expressing $m R F P$ and $m$ Citrine was carried out in submerged cultivations. Previously, our lab has used TtrpC and Ttef1 interchangeably for expression constructs as they have appeared equally efficient. Prior to the submerged cultivations, we examined if it would be more appropriate to employ common terminators between $\mathrm{P} h 4 \mathrm{~h} 3$ reporters and control promoters. Interestingly, exchanging the TtrpC for Ttef1 for the control PgpdA $A_{0.8}-m R F P$ expression resulted in a significant increase in relative expression, by a factor of $1.8(\mathrm{P}<0.01)$ (Additional file 4: Figure S5). Since most of the reference promoter constructs employed TtrpC for mRFP expression, a construct of the A. nidulans $\mathrm{P} 4 h \mathrm{~h} 3$ expression $\mathrm{mRFP}\left(\mathrm{P} h 4^{\prime}\right)$ with the TtrpC was applied (strains NID2376-78).

\section{Quantitative determination of promoter strength during submerged cultivations}

The expression profile from $\mathrm{P} h 4 h 3$ (NID) at different growth phases during submerged cultivation was evaluated by growing the strains for up to $72 \mathrm{~h}$, with sampling for RNA extraction and biomass concentration at five time-points; 6, 12, 24, 48, and $72 \mathrm{~h}$. For each time-point, the relative expression levels of $m R F P$ and $m$ Citrine were determined by RT-qPCR, and the biomass concentration determined by dry weight (DW) measurements (Fig. 5).

Based on these results (Fig. 5), Ph4h3 was most active during the early exponential growth phase, up to $12 \mathrm{~h}$, and then declined and followed the drop in biomass during onset of the stationary phase. The relative expression levels of $m R F P\left(\mathrm{Ph} 4^{\prime}\right)$ and mCitrine $\left(\mathrm{Ph} 3^{\prime}\right)$ at each time-point were similar, and did not display significant differences.

The reference strains expressing $m R F P$ from PgpdA and Ptef1 were also included in this analysis, and the relative expression from these are shown in Additional file 4: Figure S6. Regardless of the promoter construct, no difference in biomass concentrations during submerged 


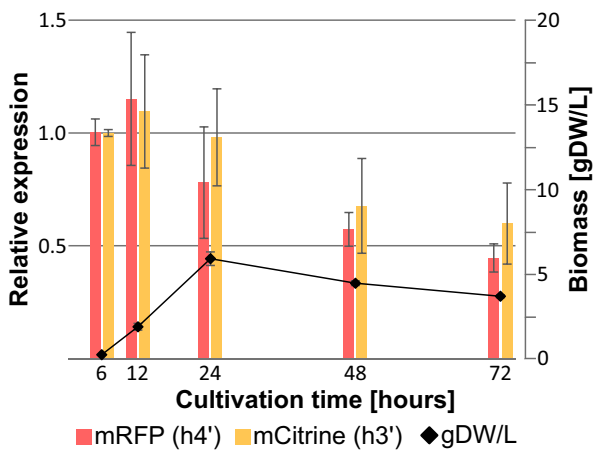

Fig. 5 Relative expression from Ph4h3 during submerged cultivation. The relative expression level (left $y$-axis) from A. nidulans Ph4h3 expressing $m R F P\left(P h 4^{\prime}\right.$, red) and $m$ Citrine (Ph $3^{\prime}$, yellow), with comparison to the biomass concentration in $\mathrm{gDW} / \mathrm{L}$ (black) (right $y$-axis) as a function of cultivation hours ( $x$-axis). After $24 \mathrm{~h}$, the media was depleted of glucose, followed by the onset of stationary phase

growth was observed, indicating that applying the endogenous Ph4h3 in A. nidulans for heterologous expression did not affect growth rate compared to using the PgpdA or Ptef1 (Additional file 4: Figure S7a). Normalization of the relative expression levels to the biomass concentration resulted in a similar pattern for the promoters, Ph4h3, PgpdA, and Ptef1 (Additional file 4: Figure S7bd), namely highest during early exponential phase $(6 \mathrm{~h})$ and declining towards the onset of stationary phase (12, $24 \mathrm{~h}$ ), and a steady low expression level during stationary phase $(48,72 \mathrm{~h})$. This may be related to the cell-cycle independent basal expression of core histones in yeast [28].

Based on the encouraging results of expression levels from Ph4h3 during both solid and submerged cultivations, we then applied multiple Ph4h3s for single locus expression of a putative BGC.

\section{Heterologous expression of the malformin biosynthetic pathway}

To evaluate the potential of applying Ph4h3 promoters for expression of a multi-gene pathway, the putative BGC responsible for production of malformins in Aspergillus brasiliensis was heterologously expressed in A. nidulans. Malformins are bicyclic pentapeptides with a disulfide bond produced by members of Aspergillus section Nigri and the biosynthesis of malformin in A. brasiliensis was recently attributed to the non-ribosomal peptide synthase (NRPS) MlfA [21]. The NRPS MlfA from A. brasiliensis synthesizes various malformin compounds that differ in the amino acid composition; malformin C, malformin A2, and at least one more uninvestigated malformin [21]. MlfA from A. brasiliensis predominantly synthesizes the compound malformin $\mathrm{C}$, which has shown potential as a pharmaceutical agent by potentiating anti-cancer drugs [36], and it displays cytotoxicity in a broad range of organisms [37].

We predict that the core BGC in A. brasiliensis consists of the gene encoding the NRPS, $m$ lf $A$ (Aspbr1_34020), and four genes coding for three transporters (mlfB: Aspbr1_186232, mlfC: Aspbr1_134974, mlfD: Aspbr1_199881) and one putative thioredoxin reductase (mlfE: Aspbr1_161173) [21]. Note that two gene models exist for $m l f C$, therefore two parallel vectors of the residual BGC, rBGC and $\mathrm{rBGC}^{*}$, were constructed; Aspbr1_134974 (mlfC) and the shorter Aspbr1_334277 $\left(m l f C^{*}\right)$.

The proposed BGC responsible for malformin synthesis was introduced in two steps. In the first step, the four hypothesized gene-cluster members $m l f B-E$ were placed under control of the Ph3h4 from A. niger and A. clavatus, whereas the second step was integration of $m l f A$ under control of $\operatorname{Pg} p d A$. We speculated that $m l f B-D$ were important in malformin production as previous deletions in the native host $A$. brasiliensis resulted in extremely poor growing colonies (unpublished data), while $m$ lfE could be involved in formation of the disulfide bond in malformin. The four genes were amplified as full genes extended with approximately $400 \mathrm{bp}$ of their native $3^{\prime}$ untranslated region as terminator sequence and using the two Ph4h3 promoters they were inserted into a combined plasmid for single-locus integration in A. nidulans integration site 4 (IS4, Fig. 6). Integration of this residual gene cluster construct (both rBGC and rBGC*) was evaluated by diagnostic PCRs from gDNA (see Additional file 4: Figure S3). All transformants displayed the same phenotype as the reference strain, showing that introducing the two heterologous Ph4h3s and $m l f B-E$ did not affect growth negatively (Additional file 4: Figure S4c-d).

The chemical analysis of rBGC strains after seven days growth at $37{ }^{\circ} \mathrm{C}$ on $\mathrm{MM}$ revealed no changes in the chromatogram compared to reference strain, and no malformin could be detected (data not shown). To reuse the pyrG marker for subsequent transformation and integration of the $m l f A$ expression construct, conidia were plated on MM supplemented with 5-FOA, and the resulting 5-FOA resistant colonies were analyzed for the presence of pyrG (Additional file 4: Figure S3d). The pyrG marker was reused for the secondary integration event to minimize metabolic changes in transformants that may affect the phenotypic readout.

The full-length $m l f A$ was assembled from four PCR fragments by USER ${ }^{\mathrm{TM}}$ cloning as described previously [21]. However, in this case we used a vector harboring target sequences for integration in $A$. nidulans IS1 [38]. Successful integration of the PgpdA-mlfA-TtrpC construct was evaluated by diagnostic PCRs from gDNA 


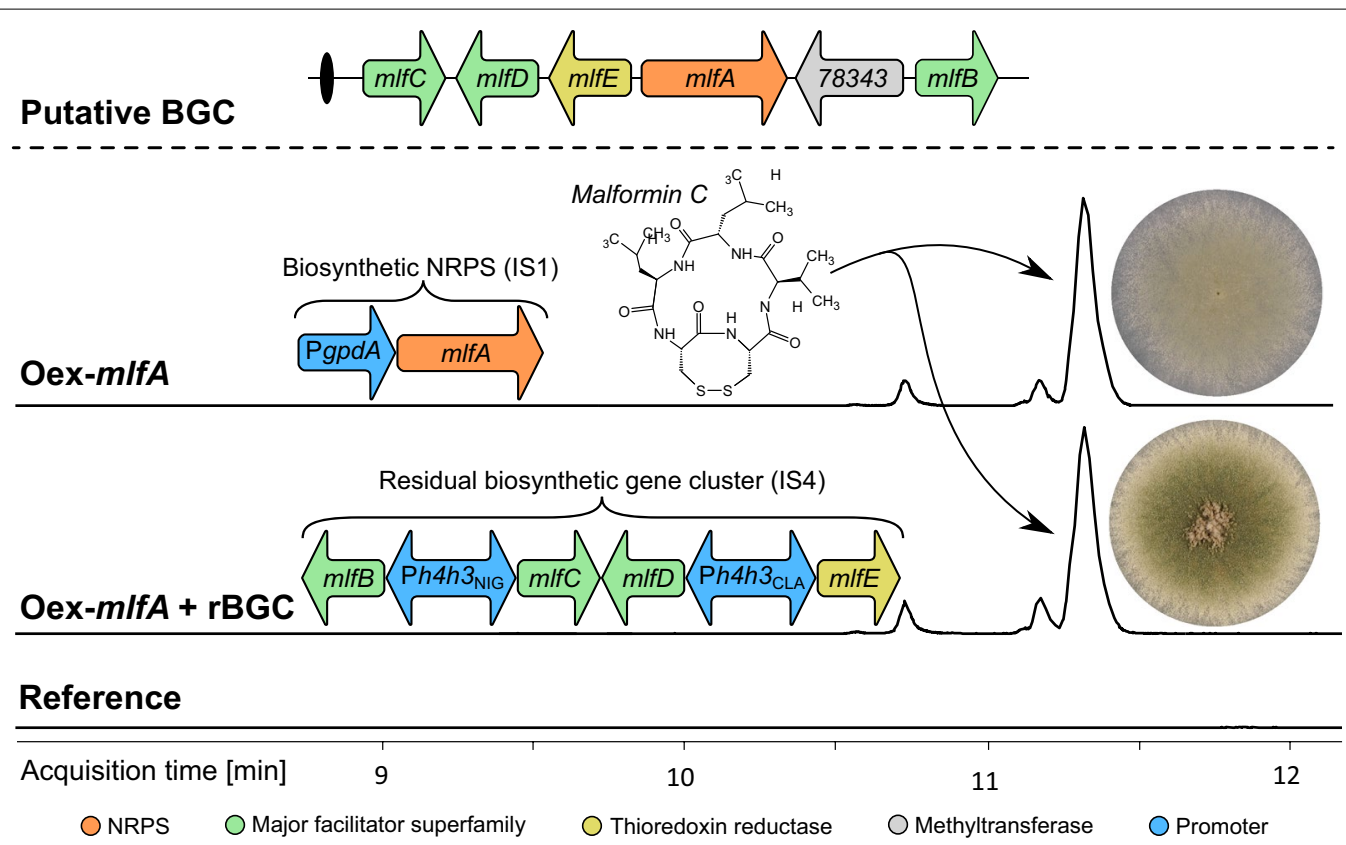

Fig. 6 Chemical analysis of malformin producing strains. The putative malformin producing BGC is conserved between producing species in Aspergillus section Nigri and the BGC organization is shown in the top of the figure. For the chemical analysis, three ElCs illustrate the malformin-production capabilities in three individual strain types. The top panel shows the expression of $m$ IfA (Oex-mIfA) alone is sufficient to produce malformin although the production comes at a price of reduced fitness and sporulation. In the middle, the residual part of the putative BGC (rBGC or rBGC*) has been integrated, and production remains at the same level as in the top, however the phenotype has been restored to normal for both constructs. Bottom panel shows that the reference strain of $A$. nidulans do not produce malformin, as it does not possess the malformin BGC. In the bottom of the figure, the enzyme types representing the genes within the BGC are listed with color codes matching the gene arrows

(Additional file 4: Figure S3). The reference host strains as well as the two strains expressing rBGC and $\mathrm{rBGC}^{*}$ were transformed by linear gene-targeting constructs and three transformants were validated by diagnostic PCR. Strikingly, the strains lacking either of the $m l f B-E$ cassettes displayed an impaired mycelial growth and sporulation phenotype showing the toxicity of malformins in $A$. nidulans (Additional file 4: Figure S4b), whereas presence of either of the two $m l f B-E$ cassettes resulted in the healthy reference phenotype as without $m$ lf $A$ (Additional file 4: Figure $S 4 \mathrm{c}-\mathrm{d}$ ).

The verified $m l f A$ overexpressing strains in the three genetic backgrounds were subjected to chemical analysis after 7 days growth at $37{ }^{\circ} \mathrm{C}$ on $\mathrm{MM}$, and these data showed that all three strategies led to production of malformins. Thus, the integration of $m l f A$ alone in the reference strain was sufficient to synthesize malformins in $A$. nidulans. The relative distribution in amounts of malformin $\mathrm{C}$ and $\mathrm{A} 2$ were similar to the native producer $A$. brasiliensis [21]. The presence of the putative thioredoxin reductase $(m l f E)$ did not affect the relative amounts of oxidized and reduced forms of the disulfide bond in any of the detected malformin compounds.
The Ph4h3 of A. niger and A. clavatus had 59\% homology with minor conserved stretches (36 nt, $25 \mathrm{nt}$, and below), limiting the risk of genetic instability and recombination between the promoters in the locus. However, we still investigated whether the locus remained intact during cultivation. Firstly, strains harboring the rBGC and $\mathrm{rBGC}^{* *}$ (prior to integration of $m l f A$ ) were propagated over a 15-day period by four times transferring spores from the peripheral zone of the colony to fresh plates, and gDNA purification from the final colonies. As expected, diagnostic-PCR analysis confirmed that the size of the expression construct was correct, with no indication of spontaneous deletions, e.g. looping out (Additional file 4: Figure S3). Secondly, strains expressing the rBGC with and without the mlfA in IS1 were propagated as single point inoculations in the center of a standard $9 \mathrm{~cm}$ petri dish with MM. Each week, the periphery of the strains had extended to the edge of the plate from where conidia were picked and transferred in the same manner to another plate. After seven successive transfers, all strains showed parental phenotypes and analysis showed that the strain still expressed the full BGC and produced malformin. 


\section{Discussion}

In this study, we aimed at demonstrating the applicability of the intergenic region between the two genes encoding histones H4.1 and H3 from multiple species of Aspergillus as a potential strong heterologous bidirectional promoter. Moreover, the goal was to address the relevance of such promoters for the stable and uniform expression of a BGC from a single locus. The use and characterization of promoters of histone-encoding genes have been reported previously in literature for steady and stable expression of, for example, selective markers and heterologous proteins [15-17]. Moreover, the genes $h 4.1$ and $h 3$ are highly and consistently expressed in both $A$. nidulans and $A$. niger at different $\mathrm{pH}$, in both defined minimal and complex media, and during solid and submerged cultivations (personal communication Mikael R. Andersen).

We showed that the Ph4h3 from A. nidulans, $A$. niger, A. flavus, A. clavatus, and A. terreus, representing five sections of the genus Aspergillus, all were functional in A. nidulans. Based on a qualitative assessment of a biological triplicate, mRFP and mCitrine displayed a simultaneous and similar intensity under the control of all the five Ph3h4s tested in freely extending leading-hyphae on agar microscopy slides, comparable to the levels of standard and popular fungal promoters PgpdA and Ptef1. This observation was also the overall conclusion from RTqPCR analysis of mRNA harvested from actively growing and sporulating colonies from solid media cultivations.

Among the five validated Ph4h3s, the promoter from A. clavatus yielded the lowest relative expression levels in $A$. nidulans. When comparing this result to the phylogenetic analysis, i.e. the dendogram (Additional file 2: Figure S1) and the underlying distance matrix (Additional file 5: Table S2), the Ph4h3 of A. clavatus also display the highest distance to the Ph4h3 of A. nidulans (0.368). A lower distance may indicate functionality due to sequence similarity between the heterologous Ph4h3 and that of the host. However, the Ph4h3 of $A$. terreus displays the second highest difference to Ph4h3 of $A$. nidulans (0.363) and obtained among the highest expression levels. As such, the distance matrix value alone cannot predict the promoters efficiency in a heterologous Aspergillus host. Of the $85 \mathrm{Ph} 4 h 3$ sequences collected in this study, 60 promoters have a distance lower or equal to that of $A$. clavatus. The experimental validation of all these promoters in other commonly applied Aspergillus species, and correlating the expression levels to the phylogenetic distance, may provide a significant addition of Aspergillus promoters.

The submerged cultivations showed that histoneexpressing promoters are strongest in the exponential growth phase, and expression drops towards the onset of the stationary phase, and is minimally expressed in stationary phase. This is not surprising since cell division stops in stationary phase, inferring a lower requirement for histones. On the other hand, histone promoters are very useful for growth-coupled production either in highdensity batch fermentations or, even more significantly, in continuous cultivations where a steady, high and constant expression for Ph4h3s can be achieved. Moreover, the bidirectional Ph $4 h 3$ promoter is constitutively active during both solid state and submerged cultivation.

The use of Ph4h3 for gene expression is limited to actively proliferating cell types, since the Ph $4 h 3$ is inactive in dormant tissues, e.g. conidia. For instance, in an $A$. nidulans $w A \triangle y A \Delta$ deletion strain displaying white conidia, we used the endogenous $\mathrm{Ph} 4 h 3$ to express the genes $w A$ and $y A$ from $A$. nidulans, which are responsible for yellow and green conidial pigmentation, respectively. This did not result in yellow or green conidia, but only secretion of yellow pigment (unpublished data). As such, the Ph4h3 was inactive in the dormant conidia, in agreement with the null requirement for further histone proteins, while being active in the growing fungal colony.

The divergent orientation of histone genes $h 4.1$ and $h 3$ was found beyond Aspergillus and is observed throughout the fungal kingdom, for example in S. cerevisiae, $N$. crassa, P. rubens, T. reesei, and basidiomycete $A$. bisporus. In addition to applying $\mathrm{P} h 4 \mathrm{~h} 3$ across sections of genus Aspergillus, we have successfully used the Ph4h3 of $A$. nidulans for production of mRFP and mCitrine in Penicillium brevicompactum (unpublished), thus highlighting the expansion in potential usage of these promoters across genera, and maybe even phyla. Strikingly, the promoter length varies from $230 \mathrm{bp}$ in $A$. bisporus to $2137 \mathrm{bp}$ in $N$. crassa (Additional file 1: Table S1), which were the minimum and maximum lengths observed in this study. Such variation merits investigations into cross-genera functionality of Ph4h3s and the common and genusspecific regulatory DNA motifs. Whether these Ph4h3s of non-Aspergilli species will display sufficient transcriptional activity within their related species, or even in Aspergillus, remains to be seen.

The regions of low conservation in Aspergilli Ph4h3, combined with the observation of a short Ph4h3 from A. bisporus, prompted us to truncate the Ph4h3 for several purposes. One being that smaller bio-blocks are preferred in synthetic biology. It is easier to amplify smaller fragments and amplify multiple assemblies of smaller fragments in, for example, sub-cloning steps. Another aim was that potentially an even stronger promoter could be created if the cell-cycle dependent regulation was lost. However, the results obtained in this study showed that arbitrary truncations of the central part in the intergenic region did not improve the expression of genes in either direction. The shortened promoters tested either lost 
some or all activity. This observation is backed by the fact that we observed highly conserved sequence regions scattered across the entire span of the promoter region. This include the Spt10 motifs of which the inner most of each pair was deleted by the $600 \mathrm{bp}$ internal truncation, which led to lack of transcriptional activity, potentially due to the loss of Spt10 activation [25].

Construction of an optimized HMM model based on the Ph4h3 sequences from 85 Aspergillus species showed 790 conserved positions suggesting a minimal promoter could be made from the consensus 790 bps. Potentially, such a promoter would display a broad and equivalent activity level in various heterologous hosts of Aspergillus. The bioinformatics analysis pinpointed highly conserved regions of the Ph4h3 sequence across 85 Aspergilli, and conserved putative motifs of TFs known to regulate histone gene expression in other organisms, including Spt10, Hap3/5 and CreA. The DNA motifs discovered in Ph4h3s of Aspergilli, could partially be found in the Ph4h3 of the other fungal species $N$. crassa, P. rubens, T. reese $i$, and $A$. bisporus. This could suggest that the Ph4h3s might be able for heterologous usage in other genera with some degree of promoter activity. As previously noted, the Ph4h3 of A. nidulans is functional in P. brevicompactum, which is partly supported by the shared DNA motifs. In S. cerevisiae, expression of core histone encoding genes occurs primarily during S-phase through DNA binding of Spt10 and recruitment of S-phase-specific Spt21 [27] and is likewise cell-cycle dependent in Aspergillus [12]. The presence of putative Spt10 binding sites in Ph $4 h 3$ and orthologues of both Spt10 and Spt21 in Aspergillus species (data not shown), along with identification of putative Hap3/5 binding sites and NEG motifs, potentiates shared regulatory features in histone gene expression across phyla. The predicted motifs could be investigated by mutagenesis studies and pulldown-assays to examine the associated transcription factors and their role in gene regulation as activator or repressors, and as potential cell-cycle responsive elements. This might provide valuable clues into the regulation of expression of core histone genes in filamentous fungi. Collectively, if miniaturized bidirectional fungal promoters are the goal, this knowledge of functional or conserved motifs/domains might be applied to shuffle the promoter sequence elements, logically or randomly, in the aspiration of generating a synthetic promoter library, as successfully conducted for $P$. pastoris by Vogl et al. [18].

The heterologous expression of mlfA from A. brasiliensis was sufficient for production of different malformins in $A$. nidulans, with the same distribution of different malformins as the native host, albeit with a stressed and growth-impaired phenotype. This showed that malformin production is toxic to A. nidulans, which in itself is not surprising since malformin has been reported to be toxic to a broad range of organisms [37]. However, co-expression of the putative residual BGC, composed of three transporters and a putative thioredoxin reductase, restored the healthy parental phenotype while producing malformin. This strongly indicates that the rBGC encodes resistance factors against the toxicity of malformin, which may be supported by the fact that the organization within the BGC is conserved between the malforminproducing Aspergilli. The presence of three genes encoding transporter proteins points to a compartmentalized biosynthesis facilitating controlled production and efficient secretion. Of the four genes from the $\mathrm{BBGC}$, only one of the predicted gene products, MlfB, had a homolog in $A$. nidulans (AN5329). This may explain why the $m l f A$ overexpression strain could survive, while the deletion of $m l f B$ in $A$. brasiliensis never resulted in viable colonies. The inclusion of the putative thioredoxin reductase did not affect the relative levels of reduced and oxidized forms of malformins during heterologous expression in $A$. nidulans, it could potentially facilitate disulfide bond formation in other target compounds in the native host. The resistance mechanisms and functions of each of the three transporters and the thioredoxin reductase warrant further investigations. Malformins are pentapeptides, yet MlfA contains four adenylation and five condensation domains [21]. The ability of $A$. nidulans to efficiently produce the same malformin types as the endogenous producer strongly indicates that MlfA is iterative in the use of one of its adenylation domains, and at that least one domain is promiscuous in the amino acid selection. With the discovery of the biosynthetic NRPS MlfA [21] and initial investigations in the BGC functions presented here, the stage is set for further studies and optimizations in malformin production, and ultimately the establishment of a fungal cell factory geared for production of this valuable compound family.

To the best of our knowledge, there has been no previous reports on the use of bidirectional heterologous Ph4h3s for expression of BGCs. The integration of two heterologous Ph4h3s in A. nidulans did not affect growth or morphology of the transformants. This indicates that sufficient amounts of regulatory proteins are present to support functional production levels of histones H4.1 and $\mathrm{H} 3$ from the endogenous Ph4h3. Often BGCs contain more than five genes, and may have up to 25 genes as in sterigmatocystin/aflatoxin biosynthesis [39]. In this extreme case, a controlled expression with multiple $\mathrm{Ph} 4 \mathrm{~h} 3 \mathrm{~s}$ in the genome may have a limit, where it influences growth, but this remains to be determined. To facilitate expression of larger gene clusters, one may envision combinatory usage of Ph4h3s with the self-cleaving A2 peptide for polycistronic expression [40], which was 
elegantly applied for expression of four biosynthetic genes in $A$. nidulans from a single promoter [41]. The use of Ph4h3s for uniform expression of transporters and tailoring enzymes could also be practical when the synthase or synthetase expression is regulated by a tunable promoter, so the pool of enzymes are available for production when the main scaffold is produced.

The equivalent high expression levels from the investigated Ph4h3s may be disadvantageous for differential expression of pathway enzyme encoding genes and hereby optimizing production levels. Bidirectional promoters developed for P. pastoris by Vogl et al. [18] collectively displayed a 61-fold range of expression levels, thereby enabling optimization of the four pathway genes expressed. Such optimizations are also possible in filamentous fungi, e.g. using Ph4h3s in combination with a weaker mono- or bidirectional promoters. For this approach, promoter strengths always should be benchmarked against a standard promoters as presented here and elsewhere, e.g. $p c b A B / p c b C$ from $P$. chrysogenum [42].

Using constitutive strong heterologous monodirectional promoters in same manner as presented with the Ph4h3s would be possible as long as the heterologous promoters have similar expression profiles in the host. For one-locus BGC expression, it will depend on the sequence homology regarding genetic stability. In future applications, the use of heterologous promoters such as Ph4h3 in a single locus will be a balance of choosing the strongest options while keeping the sequence identity of the multiple Ph4h3s low enough, e.g. less than $90 \%$ identity, as issues of genetic instability may arise from single locus integration of multiple Ph4h3s.

\section{Conclusion}

In Aspergillus, the intergenic region of histone genes $h 4.1$ and $h 3$ has proven to be a strong constitutive bidirectional promoter (Ph4h3) with equivalent expression levels from the two ends. Expression levels from heterologous Ph4h3s in A. nidulans are comparable to that of the endogenous Ph4h3, PgpdA and Ptef1 during solid-state cultivation. The $\mathrm{P} h 4 h 3$ is active during submerged cultivation with an expression pattern similar to that of PgpdA and Ptef1 at the different growth stages. The Ph4h3s from genus Aspergillus contain a number of conserved DNA motifs, which likely have regulatory functions. Two heterologous Ph4h3s were applied for genetically stable single-locus integration of four genes from the malformin biosynthetic pathway of A. brasiliensis. The heterologous expression of $m l f A$ alone was sufficient for malformin production in $A$. nidulans indicating that one of its four adenylation domains is iterative. However, expression of the entire putative BGC offers the heterologous host protection from the toxicity of malformin.

\section{Methods}

\section{Strains and cultivations}

The A. nidulans strain NID1 (argB2, pyrG89, veA1, $n k u A \Delta)$ [43], was used as host for all expression-cassette integrations. Genomic DNA (gDNA) from NID1, A. niger (ATCC1015), A. clavatus (NRRL1), A. flavus (NRRL 3357), and $A$. terreus (NIH2624), and $A$. brasiliensis (CBS101740) was isolated using FastDNA SPIN Kit for Soil DNA extraction kit (MP Biomedicals, USA). All strains employed and created in this study are listed in Additional file 6: Table S7. Escherichia coli strain DH5 $\alpha$ was used as host for plasmid propagations.

All strains were cultivated using solid and liquid glucose based minimal medium (MM) (1\% w/v) glucose, $1 \times$ nitrate salt solution [44], $0.001 \%(\mathrm{w} / \mathrm{v})$ thiamine, $1 \times$ trace metal solution [45], 2\% (w/v) agar for solid medium. Media was supplemented with $10 \mathrm{mM}$ uridine (Uri), $10 \mathrm{mM}$ uracil (Ura), and $4 \mathrm{mM} \mathrm{L}$-arginine (Arg) when required. Solid $\mathrm{MM}+\mathrm{Arg}+\mathrm{Uri}+\mathrm{Ura}$ plates containing 5 -fluoroorotic acid (5-FOA, $1.3 \mathrm{mg} / \mathrm{mL}$ ) were used in counter-selecting pyrG. For transformation media (TM), glucose was replaced with $1 \mathrm{M}$ sucrose.

\section{PCR for fragment production and strain verification}

PCR was used for production of fragments for USER ${ }^{\mathrm{TM}}$ cloning and strain validation, and primers are listed in Additional file 6: Table S8. Fragments for $\mathrm{USER}^{\mathrm{TM}}$ cloning were amplified using the Phusion U Hot Start PCR Master Mix (Thermo Scientific) and $0.4 \mu \mathrm{M}$ primers (Integrated DNA Technologies, IDT), $<10$ ng gDNA or $20 \mathrm{ng}$ plasmid. PCR for strain verification used standard $50 \mu \mathrm{L}$ PCR reactions including; $1 \mathrm{U}$ PfuX7, $1 \times$ Phusion HF buffer (New England Biolabs, USA), $0.2 \mathrm{mM}$ dNTPs, $0.4 \mu \mathrm{M}$ primers, $<10 \mathrm{ng}$ gDNA. All reactions were performed on a T100 Thermal Cycler (Bio-Rad) with initial denaturation $\left(98{ }^{\circ} \mathrm{C}\right.$ for $5 \mathrm{~min}$ ) followed by 35 cycles $\left\{98{ }^{\circ} \mathrm{C}\right.$ for $30 \mathrm{~s} ; 58-62{ }^{\circ} \mathrm{C}$ for $30 \mathrm{~s}$ (depending on the primer pair), $72{ }^{\circ} \mathrm{C} 1-5 \mathrm{~min}$ (using $1 \mathrm{~kb} / \mathrm{min}$ ) $\}$ and final elongation $\left(72{ }^{\circ} \mathrm{C}\right.$ for $\left.10 \mathrm{~min}\right)$.

\section{Vector construction}

All plasmids are listed in Additional file 6: Table S9 and two vectors; pAC223 (pU2005-4) and pAC1190 (pU2005-1) served as basis for USER vector constructions [38] employing the ampicillin resistance marker and $E$. coli oriC. The fungal part of the vectors contain: selection marker pyrG (orotidine-5'-phosphate decarboxylase) from $A$. fumigatus under control of its native promoter and terminator flanked by a direct repeat of 282 bp [46]; a PacI/Nt.BbvCI cassette for USER cloning 
mediated expression cassette insertion; two $1 \mathrm{~kb}$ targeting sequences for integration sites 1 [38] and 4 [34] in pAC1190 and pAC223, respectively. The assembled genetargeting substrates were flanked by SwaI sites for linearization. The A. nidulans IS4 is located on chromosome II in the intergenic region of AN4252 and AN4251 based of high transcriptional activity of the neighboring genes [34].

The Ph4h3 of A. nidulans (FGSC A4) was defined as the intergenic region between the translational start sites (ATG) of the genes encoding histone H4.1 (AN0734.2) and H3 (AN0733.2). The homologs in the other species were identified by performing a BLASTp search [22] for homologous proteins. The complete list of the histone encoding genes and the intergenic $\mathrm{P} h 4 h 3$ sequences applied in this study is provided in Additional file 1: Table S1. Plasmids harboring gene cassettes of $\mathrm{P} h 4 h 3$ and reference promoters expressing $m R F P 1$ [47] and $m$ Citrine [48], and the malformin BGC were generated by amplifying PCR fragments and USER cloning according to the scheme and functionalities presented in Additional file 6: Table S10.

For the heterologous expression of the malformin BGC in $A$. nidulans it should be noted that the Ph4h3 from $A$. niger and $A$. clavatus were used to build the expression cassette for the rBGC of three putative major facilitator superfamily (MFS) transporters; mlfB (Aspbr1_186232, mlfC (Aspbr1_134974), and mlfD (Aspbr1_199881), and one putative thioredoxin reductase mlfE (Aspbr1_161173). All genes included their native terminators (estimated at $\sim 400$ bp length) and had $5^{\prime}$ overhangs matching the Ph4h3 of $A$. niger ( $m l f B$ and $m l f C$ ) and A. clavatus ( $m l f D$ and $m l f E$ ), see Fig. 6 . Due to their native head-to-head orientation, $m l f C$ and $m l f D$ were amplified as single fragment. Note that two gene models exist for $m l f C$, therefore two parallel vectors, $\mathrm{rBGC}$ and rBGC*, were constructed; Aspbr1_134974 ( $\mathrm{mlfC})$ and the shorter Aspbr1_334277 ( $\left.m l f \mathrm{C}^{*}\right)$.

The vector containing $m l f A$ (Aspbr1_34020) was constructed as described in Theobold et al. [21], but specifically $m l f A$ was under control of the $2.3 \mathrm{~kb} g p d A$ promoter and $\operatorname{trp} C$ terminator of $A$. nidulans, with $1 \mathrm{~kb}$ targeting sequences for $A$. nidulans IS1 [38].

\section{Promoter sequence alignment}

All fungal genome sequence data retrieved from the Joint Genome Institute (genome.jgi.doe.gov) [49] and AspGD (aspgd.org) [50]. Homologous promoter sequences were identified as described in previous section for 85 Aspergillus species and 5 non-Aspergillus fungi ( $N$. crassa, P. rubens, T. reesei, A. bisporus and both copies from S. cerevisiae). The DNA for these $91 \mathrm{Ph} 4 \mathrm{~h} 3$ regions was extracted for further sequence analysis. Initial multiple sequence alignments (MSA) were performed using Clustal W (version 2.1) [51] and showed that the nonAspergillus species shared only very limited sequence conservation. For this reason, only the 85 Aspergillus sequences were used to define the $\mathrm{P} h 4 h 3$ alignment. An initial Clustal W alignment was used to fit a hidden Markov model (HMM) using HMMER (version 3.2.1) [52]. As the $5^{\prime}$ and $3^{\prime}$ ends of the alignment were not conserved for a number of species, but were included in the HMM, an iterative refinement was performed to identify the start and end of the conserved region. The initial HMM with 835 positions was used to re-align the 85 sequences using hmmalign and the '-trim' option. This was repeated until the consensus sequence converged (after 15 iterations) resulting in an optimized 790 position HMM. This HMM was then visualized as a DNA sequence logo using Skylign [53]. The consensus sequence from the optimized HMM was extracted using 'hmmemit -C -minl 0.25 -minu 0.5'.

\section{Genetic transformation}

Protoplast formation and transformation was done as previously described [46, 54]. Transformation into NID1 protoplasts was conducted for all gene-targeting constructs intended for expression of fluorescent proteins, $m$ lff, or the rBGC and $\mathrm{rBGC}^{*}$ constructs. Three individual and double resteaked transformants for each construct were verified by rigorous diagnostic PCR on purified gDNA, validating the targeted integration and testing for homokaryons and heterokaryons (Additional file 4: Figure S3). This was possible with primer pairs amplifying either an intact integration site or disrupted locus due to the integration of the gene-expression cassette.

The gene-targeting substrates based on rBGC and rBGC$^{*}$ were integrated in A. nidulans IS4 in NID1. Two transformants of each type were validated by PCR employing purified gDNA genotyping (Additional file 4: Figure S3a-c), and one of each was subsequently cultivated on 5-FOA for recycling of the pyrG marker (Additional file 4: Figure S3d). Protoplasts of these strains, named $\mathrm{rBGC}$ and $\mathrm{rBGC}^{*}$, respectively, were transformed with the mlfA expression construct and validated (Additional file 4: Figure S3). All strains expressing genes from the malformin BGC were analyzed by chemical extraction, as described previously [21].

RNA extraction, cDNA synthesis, RT-qPCR and data analysis RNA was extracted, using the RNeasy Plus Mini Kit (Qiagen), from biomass harvested from 3-day cultivations on solid medium and $6,12,24,48$ and $72 \mathrm{~h}$ submerged cultivations. The cDNA was synthesized using the SensiFAST cDNA Synthesis Kit (Bioline) according to manufacturer's 
recommendations. RT-qPCR conducted using the SensiFAST SYBR No-ROX Kit (Bioline) according to manufacturer's recommendations, on a CFX Connect (BioRad) using the software BioRad CFX Manager 3.1, and white 96 well RT-qPCR plates and clear lids (VWR International). RT-qPCR was conducted using primers targeting mCitrine (P71 + P72; 280 bp), mRFP (P73 + P74; 133 bp), and actin-encoding gene actA (AN6542) (P75+ P76; $132 \mathrm{bp}$ ). Active splicing the actA mRNA was confirmed by gel electrophoresis by determining an introns-free band of $132 \mathrm{bp}$ compared to the gDNA band of $189 \mathrm{bp}$. RT-qPCR data was analyzed according to the method described by Pfaffl 2001 [55]. Reaction efficiencies were determined using cDNA from a single strain at five concentrations $(0.5,2.5,12.5,64$, and 320 ng per $20 \mu \mathrm{L}$ reaction) and calculating the slope between the Ct-values and the $\log 10$ of the cDNA concentration [55]. The reaction efficiencies calculated and applied were: 1.982 (act $A$ ), 1.999 (mRFP), and 1.948 (mCitrine). Statistical analysis was performed using a $t$-test with two-tailed homoscedastic distribution, calculated from the $\Delta \Delta$ Ct-values. All error bars represent the standard error of the mean.

\section{Fluorescence microscopy}

Fresh spores suspensions $\left(10 \mu \mathrm{L}\right.$ of $\sim 10^{5}$ spores $\left./ \mathrm{mL}\right)$ were inoculated on glass slides with $0.5 \mathrm{~mL}$ solid MM $(1 \%$ agar $)+$ Arg and incubated for $\sim 20 \mathrm{~h}$ in petri dishes in micro-perforated bags at $37{ }^{\circ} \mathrm{C}$. A cover slide and immersion oil were applied and photos taken with a $100 \times$ objective and exposure times $200 \mathrm{~ms}$ (normal filter) or $750 \mathrm{~ms}$ (YFP and RFP filters), using a Nikon Eclipse E1000 fluorescence microscope equipped with a QImaging Retiga Exi camera.

\section{Supplementary information}

Supplementary information accompanies this paper at https://doi. org/10.1186/s40694-019-0088-3.

Additional file 1. Identification of Ph4h3. Table S1. Identification of histone $h 4.1$ and $h 3$ genes in 85 Aspergillus species and reference fungi by BLASTp of A. nidulans $\mathrm{H} 4.1$ and H3. Curated usage of gene models and prediction of the Ph4h3 sequence.

Additional file 2. Ph4h3 sequence analysis. Figure S1. Dendogram of Ph4h3 sequences from 85 Aspergilli. Figure S2. Logo plot of the alignment of Ph4h3 from 85 Aspergilli.

Additional file 3. Bioinformatic analysis of DNA motifs. Table S3. All results of JASPAR analysis. Table S4. Results of JASPAR analysis with score above 9. Table S5. Best hit of YeTFaSCo Spt10 in each of the 85 Ph4h3 sequences. Table S6. Best eight hits of YeTFaSCo Spt10 in the consensus Ph4h3 sequence. Additional motif results JASPAR analysis in reference fungi.

Additional file 4. Supplementing experimental data. Figure S3. PCR validation strategy. Figure S4. Pictures of fungal colonies. Figure S5. Relative expression from promoters during solid-state cultivation. Figure S6. Relative expression from reference promoters during submerged cultivation.
Figure S7. Biomass concentration during submerged cultivation and relative expression from promoters normalized to biomass concentration.

Additional file 5. Ph4h3 dendogram distance matrix. Table S2. The distance matrix generated from the alignment of 85 Aspergilli Ph4h3.

Additional file 6. Tables of Strains, Primers, Plasmids and USER fragments. Table S7. Strains used in this study. Table S8. Primers used in this study. Table S9. Plasmids used in this study. Table S10. USER fragments applied in this study.

\section{Abbreviations}

Ph4h3: promoter of $h 4.1$ and $h 3$; mRFP1: monomeric red fluorescent protein 1; RT-qPCR: reverse transcription quantitative polymerase chain reaction; IS: integration site; SM: secondary metabolite; NRPS: non-ribosomal peptide synthetase; BGC: biosynthetic gene cluster; rBGC: residual biosynthetic gene cluster; TF: transcription factor; JGI: joint genome institute; HMM: hidden Markov model; MSA: multiple sequence alignments; DW: dry weight; USER: uracil specific excision reagent.

\section{Acknowledgements}

Authors acknowledge Amalie G. Kloster and Karolis Petkevicius for aiding plasmid and strain construction. We thank Prof. Jens C. Frisvad for assistance with Aspergillus taxonomy and curation of species names. The vector harboring $m$ Citrine was kindly donated by R. Fischer and S. Gradia (Addgene plasmid \#29771).

\section{Authors' contributions}

JKHR and JBH conceived the study. JKHR designed and performed the experimental work. JKHR, CTW and JBH analyzed the data. JKHR, CTW and JBH wrote the manuscript. All authors read and approved the final manuscript.

\section{Funding}

This work has been supported by Innovation Fund Denmark, Grant No. 6150-00031B.

\section{Availability of data and materials}

All promoter sequences are available in Additional file 1. Majority of bioinformatics results provided in Additional files 2, 3, 5. Strains, RT-qPCR data, fluorescent images, and chemical analysis data are available upon request.

\section{Ethics approval and consent to participate}

Not applicable.

\section{Consent for publication}

The sequence data was retrieved from and produced by the US Department of Energy Joint Genome Institute http://www.jgi.doe.gov/in collaboration with the user community.

\section{Competing interests}

The authors declare that they have no competing interests.

Received: 21 October 2019 Accepted: 23 November 2019

Published online: 09 December 2019

References

1. Punt PJ, Dingemanse MA, Kuyvenhoven A, Soede RDM, Peuwels PH, van Hondel C. Functional elements in the promoter region of the Aspergillus nidulans gpdA gene encoding glyceraldehyde-3-phosphate dehydrogenase. Gene. 1990;93:101-9.

2. Kitamoto N, Matsui J, Kawai Y, Kato A, Yoshino S, Ohmiya K, et al. Utilization of the TEF1-a gene (TEF1) promoter for expression of polygalacturonase genes, pgaA and pgaB, in Aspergillus oryzae. Appl Microbiol Biotechnol. 1998;50:85-92.

3. Waring RB, May GS, Morris NR. Characterization of an inducible expression system in Aspergillus nidulans using alcA and tubulin coding genes. Gene. 1989;79:119-30. 
4. Boel E, Hansen MT, Hjort I, Høegh I, Fil NP. Two different types of intervening sequences in the glucoamylase gene from Aspergillus niger. Eur Mol Biol Organ. 1984;3:1581-5.

5. Wanka F, Cairns T, Boecker S, Berens C, Happel A, Zheng X, et al. Tet-on, or Tet-off, that is the question: advanced conditional gene expression in Aspergillus. Fungal Genet Biol. 2016;89:72-83.

6. Wiemann P, Keller NP. Strategies for mining fungal natural products. J Ind Microbiol Biotechnol. 2014;41:301-13.

7. Chiang YM, Szewczyk E, Davidson AD, Keller N, Oakley BR, Wang CCC. A gene cluster containing two fungal polyketide synthases encodes the biosynthetic pathway for a polyketide, asperfuranone, in Aspergillus nidulans. J Am Chem Soc. 2009;131:2965-70.

8. Yin WB, Baccile JA, Bok JW, Chen Y, Keller NP, Schroeder FC. A nonribosomal peptide synthetase-derived iron(III) complex from the pathogenic fungus Aspergillus fumigatus. J Am Chem Soc. 2013;135:2064-7.

9. Porquier A, Morgant G, Moraga J, Dalmais B, Luyten I, Simon A, et al. The botrydial biosynthetic gene cluster of Botrytis cinerea displays a bipartite genomic structure and is positively regulated by the putative Zn(II)2Cys6 transcription factor BcBot6. Fungal Genet Biol. 2016;96:33-46.

10. Schäpe P, Kwon MJ, Baumann B, Gutschmann B, Jung S, Lenz S, et al. Updating genome annotation for the microbial cell factory Aspergillus niger using gene co-expression networks. Nucleic Acids Res. 2019;47:559-69.

11. Gladyshev E. Repeat-induced point mutation (RIP) and other genome defense mechanisms in fungi. Microbiol Spectr. 2017;5:1-21.

12. Ehinger A, Denison SH, May GS. Sequence, organization and expression of the core histone genes of Aspergillus nidulans. Mol Gen Genet. 1990;222:416-24.

13. Eriksson PR, Ganguli D, Nagarajavel V, Clark DJ. Regulation of histone gene expression in budding yeast. Genetics. 2012;191:7-20.

14. Mariño-Ramírez L, King IK, Landsman D. Multiple independent evolutionary solutions to core histone gene regulation. Genome Biol. 2006;7:R122.

15. Belshaw NJ, Haigh NP, Fish NM, Archer DB, Alcocer MJC. Use of a histone $\mathrm{H} 4$ promoter to drive the expression of homologous and heterologous proteins by Penicillium funiculosum. Appl Microbiol Biotechnol. 2002;60:455-60

16. Suzuki S, Tada S, Fukuoka M, Taketani H, Tsukakoshi Y, Matsushita M, et al. A novel transformation system using a bleomycin resistance marker with chemosensitizers for Aspergillus oryzae. Biochem Biophys Res Commun. 2009;383:42-7.

17. Wartmann T, Bellebna C, Böer E, Bartelsen O, Gellissen G, Kunze G. The constitutive AHSB4 promoter-a novel component of the Arxula adeninivorans-based expression platform. Appl Microbiol Biotechnol. 2003;62:528-35.

18. Vogl T, Kickenweiz T, Pitzer J, Sturmberger L, Weninger A, Biggs BW, et al. Engineered bidirectional promoters enable rapid multi-gene co-expression optimization. Nat Commun. 2018;9:1-13.

19. Menne S, Walz M, Kück U. Expression studies with the bidirectional $p c b A B-p c b C$ promoter region from Acremonium chrysogenum using reporter gene fusions. Appl Microbiol Biotechnol. 1994;42:57-66.

20. Wiemann P, Soukup AA, Folz JS, Wang P-M, Noack A, Keller NP. ColN: co-inducible nitrate expression system for secondary metabolites in Aspergillus nidulans. Fungal Biol Biotechnol. 2018:5:1-10.

21. Theobald S, Vesth TC, Rendsvig JK, Nielsen KF, Riley R, de Abreu LM, et al. Uncovering secondary metabolite evolution and biosynthesis using gene cluster networks and genetic dereplication. Sci Rep. 2018;8:1-12.

22. Altschup SF, Gish W, Miller W, Myers EW, Lipman DJ. Basic local alignment search tool. J Mol Biol. 1990;215:403-10.

23. Khan A, Fornes O, Stigliani A, Gheorghe M, Castro-Mondragon JA, Van Der Lee R, et al. JASPAR 2018: update of the open-access database of transcription factor binding profiles and its web framework. Nucleic Acids Res. 2018:46:D260-6.

24. Koessler H, Kahle J, Bode C, Doenecke D, Albig W. Human replicationdependent histone $\mathrm{H} 3$ genes are activated by a tandemly arranged pair of two CCAAT boxes. Biochem J. 2004;384:317-26.

25. Eriksson PR, Mendiratta G, McLaughlin NB, Wolfsberg TG, Marino-Ramirez $\mathrm{L}$, Pompa TA, et al. Global regulation by the yeast Spt10 protein is mediated through chromatin structure and the histone upstream activating sequence elements. Mol Cell Biol. 2005;25:9127-37.
26. Osley MA, Gould J, Kim S, Kane M, Hereford L. Identification of sequences in a yeast histone promoter involved in periodic transcription. Cell. 1986;45:537-44.

27. Kurat CF, Lambert JP, Petschnigg J, Friesen H, Pawson T, Rosebrock A, et al. Cell cycle-regulated oscillator coordinates core histone gene transcription through histone acetylation. Proc Natl Acad Sci USA. 2014;111:14124-9.

28. Mei Q, Huang J, Chen W, Tang J, Xu C, Yu Q, et al. Regulation of DNA replication-coupled histone gene expression. Oncotarget. 2017:8:95005-22.

29. De Boer CG, Hughes TR. YeTFaSCo: a database of evaluated yeast transcription factor sequence specificities. Nucleic Acids Res. 2012;40:169-79.

30. Freeman KB, Karns LR, Lutz KA, Smith MM. Histone H3 transcription in Saccharomyces cerevisiae is controlled by multiple cell cycle activation sites and a constitutive negative regulatory element. Mol Cell Biol. 1992;12:5455-63.

31. Drysdale MR, Kolze SE, Kelly JM. The Aspergillus niger carbon catabolite repressor encoding gene, creA. Gene. 1993;130:241-5.

32. Bean JM, Siggia ED, Cross FR. High functional overlap between Mlul cell-cycle box binding factor and Swi4/6 cell-cycle box binding factor in the G1/S transcriptional program in Saccharomyces cerevisiae. Genetics. 2005;171:49-61.

33. Simon I, Barnett J, Hannett N, Harbison CT, Rinaldi NJ, Volkert TL, et al. Serial regulation of transcriptional regulators in the yeast cell cycle. Cell. 2001;106:697-708.

34. Holm DMK. Development and implementation of novel genetic tools for investigation of fungal secondary metabolism. Ph.D. thesis. Technical University of Denmark; 2013.

35. Punt PJ, Oliver RP, Dingemanse MA, Pouwels PH, van den Hondel CA. Transformation of Aspergillus based on the hygromycin B resistance from Escherichia coli. Gene. 1987;56:117-24.

36. Wang J, Jiang Z, Lam W, Gullen EA, Yu Z, Wei Y, et al. Study of malformin $C$, a fungal source cyclic pentapeptide, as an anti-cancer drug. PLoS ONE. 2015;10:1-19.

37. Anderegg RJ, Biemann K, Büchi G, Cushman M. Malformin C, a new metabolite of Aspergillus niger. J Am Chem Soc. 1976;98:3365-70.

38. Hansen BG, Salomonsen B, Nielsen MT, Nielsen JB, Hansen NB, Nielsen $\mathrm{KF}$, et al. Versatile enzyme expression and characterization system for Aspergillus nidulans, with the Penicillium brevicompactum polyketide synthase gene from the mycophenolic acid gene cluster as a test case. Appl Environ Microbiol. 2011;77:3044-51.

39. Yu J, Chang P-K, Ehrlich KC, Cary JW, Bhatnagar D, Cleveland TE, et al. Clustered pathway genes in aflatoxin biosynthesis. Appl Environ Microbiol. 2004;70:1253-62

40. Schuetze T, Meyer V. Polycistronic gene expression in Aspergillus niger. Microb Cell Fact. 2017;16:1-8.

41. Hoefgen S, Lin J, Fricke J, Stroe M, Mattern DJ, Kufs JE, et al. Facile assembly and fluorescence-based screening method for heterologous expression of biosynthetic pathways in fungi. Metab Eng. 2018;48:44-51.

42. Polli F, Meijrink B, Bovenberg RAL, Driessen AJM. New promoters for strain engineering of Penicillium chrysogenum. Fungal Genet Biol. 2016;89:62-71.

43. Nielsen JB, Nielsen ML, Mortensen UH. Transient disruption of nonhomologous end-joining facilitates targeted genome manipulations in the filamentous fungus Aspergillus nidulans. Fungal Genet Biol. 2008:45:165-70.

44. Kaminskyj SGW. Fundamentals of growth, storage, genetics and microscopy of Aspergillus nidulans. Fungal Genet Rep. 2001;48:25-31.

45. Cove DJ. The induction and repression of nitrate reductase in the fungus Aspergillus nidulans. Biochim Biophys Acta. 1966;113:51-6.

46. Nielsen ML, Albertsen L, Lettier G, Nielsen JB, Mortensen UH. Efficient PCR-based gene targeting with a recyclable marker for Aspergillus nidulans. Fungal Genet Biol. 2006;43:54-64.

47. Campbell RE, Tour O, Palmer AE, Steinbach PA, Baird GS, Zacharias DA, et al. A monomeric red fluorescent protein. Proc Natl Acad Sci USA. 2002;99:7877-82.

48. Griesbeck O, Baird GS, Campbell RE, Zacharias DA, Tsien RY. Reducing the environmental sensitivity of yellow fluorescent protein. J Biol Chem. 2001;276:29188-94

49. Grigoriev IV, Nordberg H, Shabalov I, Aerts A, Cantor M, Goodstein D, et al. The genome portal of the department of energy joint genome institute. Nucleic Acids Res. 2012:40:D26-32. 
50. Cerqueira GC, Arnaud MB, Inglis DO, Skrzypek MS, Binkley G, Simison $M$, et al. The Aspergillus genome database: multispecies curation and incorporation of RNA-Seq data to improve structural gene annotations. Nucleic Acids Res. 2014;42:D705-10.

51. Larkin MA, Blackshields G, Brown NP, Chenna R, Mcgettigan PA, McWilliam H, et al. Clustal $W$ and Clustal $X$ version 2.0. Bioinformatics. 2007:23:2947-8

52. Eddy SR. Accelerated profile HMM searches. PLoS Comput Biol. 2011:7:1-16.

53. Wheeler TJ, Clements J, Finn RD. Skylign: a tool for creating informative, interactive logos representing sequence alignments and profile hidden Markov models. BMC Bioinform. 2014;15:1-9.
54. Nødvig CS, Nielsen JB, Kogle ME, Mortensen UH. A CRISPR-Cas9 system for genetic engineering of filamentous fungi. PLOS ONE. 2015;10:1-18.

55. Pfaffl MW. A new mathematical model for relative quantification in realtime RT-PCR. Nucleic Acids Res. 2001;29:2002-7.

\section{Publisher's Note}

Springer Nature remains neutral with regard to jurisdictional claims in published maps and institutional affiliations.
Ready to submit your research? Choose BMC and benefit from:

- fast, convenient online submission

- thorough peer review by experienced researchers in your field

- rapid publication on acceptance

- support for research data, including large and complex data types

- gold Open Access which fosters wider collaboration and increased citations

- maximum visibility for your research: over $100 \mathrm{M}$ website views per year

At BMC, research is always in progress.

Learn more biomedcentral.com/submissions 